\title{
Incidencia de complicaciones post quirúrgicas en cirugía bucal.
}

\section{Incidence of post-surgical complications in oral surgery.}

\author{
Francisca Poblete1; Matías Dallaserra ${ }^{1,2}$; Nicolás Yanine ${ }^{1,2,3}$; Ignacio Araya ${ }^{1,2}$; \\ Ricardo Cortés ${ }^{2}$; Cristian Vergara ${ }^{2}$; Julio Villanueva ${ }^{1,2,3^{*}}$
}

\begin{abstract}
1. Departamento de Cirugía y Traumatología Bucal y Maxilofacial, Facultad de Odontología, Universidad de Chile, Santiago, Chile.

2. Centro Cochrane Unidad Odontología Basada en Evidencia, Facultad de Odontología, Universidad de Chile, Santiago, Chile.

3. Unidad de Cirugía Maxilofacial, Hospital Clínico San Borja-Arriarán. Santiago, Chile.

* Correspondencia Autor: Julio Villanueva | Dirección: Olivos 943 Independencia (8380492).

Santiago de Chile | Teléfono: +562 2978 1841|

E-mail: javm@uchile.cl

Trabajo recibido el 27/07/2019.

Aprobado para su publicación el 20/01/2020
\end{abstract}

\begin{abstract}
RESUMEN
Objetivo: El objetivo principal de este estudio fue determinar la incidencia de complicaciones post cirugía bucal. Metodología: Se desarrolló un estudio observacional, descriptivo, con el uso del registro prospectivo de la totalidad de pacientes ingresados para cirugía bucal del Complejo Hospitalario San Borja-Arriarán durante doce meses de observación (abril 2017 a marzo 2018). Resultados: La muestra estuvo conformada por 532 pacientes sometidos a procedimientos quirúrgicos de cirugía bucal y 29 casos de complicación postquirúrgica. La incidencia de complicaciones alcanzó un $5,5 \%$ y se observó de manera predominante en intervenciones de tipo exodoncia. La complicación mayormente observada fue la alveolitis alcanzando un $2,5 \%$ de las cirugías de terceros molares y un $3,7 \%$ de las exodoncias de otros dientes. Las hemorragias postoperatorias se observaron en un $1,1 \%$ de las cirugías de terceros molares. Otras complicaciones postquirúrgicas fueron abscesos de espacios faciales, parestesia del nervio alveolar inferior, hematomas, equimosis y periostitis. Conclusión: Los resultados fueron similares a los reportados en la literatura tanto en su frecuencia como en el tipo de complicación.
\end{abstract}

PALABRAS CLAVE

Cirugía bucal; Incidencia; Complicaciones postquirúrgicas; Alveolitis; Tercer molar.

Int. J. Inter. Dent Vol. 13(1); 13-16, 2020.

\begin{abstract}
Objective: The main objective of this investigation was to determine the incidence of postoperative complications in oral surgery. Materials and methods: An observational and descriptive study was developed with the use of the prospective registry of the patients admitted for oral surgery in the San Borja Arriarán Hospital Complex for a twelve-month period of observation (April 2017 to March 2018). Results: The sample consisted of 532 patients undergoing surgical procedures of oral surgery and 29 cases of postoperative complications. The incidence of complications reached $5,5 \%$ and it was observed predominantly in interventions of tooth extraction. The most commonly observed complication was dry socket, reaching $2,5 \%$ of third molar surgeries and $3,7 \%$ of extractions of other teeth. Postoperative hemorrhages were observed in $1,1 \%$ of third molar surgeries. Other postoperative complications were facial spaces abscesses, paresthesia of the inferior alveolar nerve, bruising, ecchymosis and periostitis. Conclusions: The results were similar to those reported in the literature both in their frequency and in the type of complication.

KEY WORDS
\end{abstract}

Oral surgery; Incidence; Postoperative complications; Dry socket; Third molar.

Int. J. Inter. Dent Vol. 13(1); 13-16, 2020.

\section{INTRODUCCIÓN}

La cirugía bucal y máxilo facial corresponde a la especialidad médico quirúrgica que se ocupa de la prevención, diagnóstico, tratamiento y rehabilitación de las patologías de la cara, estructuras cervicales y la cavidad bucal. La cirugía bucal corresponde a un área de esta especialidad que se ocupa de la prevención, diagnóstico y tratamiento (médico y/o quirúrgico) tanto en el aspecto funcional como estético, de las patologías congénitas y adquiridas de los tejidos blandos y duros de la boca. La cirugía bucal engloba diferentes procedimientos entre los cuales podemos mencionar las exodoncias, fenestraciones, cirugías de reborde alveolar, biopsias de la mucosa oral, entre otros. Las tasas de complicaciones de cirugía bucal varían según el tipo de procedimiento, de los cuales, la exodoncia es el que presenta una mayor incidencia ${ }^{(1)}$.

La exodoncia es uno de los procedimientos más realizados en cirugía bucal y su objetivo es remover dientes afectados con alguna patología que comprometa la salud del individuo, siendo los terceros molares los más frecuentes ${ }^{(2,3)}$. La extracción puede ser un procedimiento simple o complejo y esto dependerá de los factores que afectan su remoción ${ }^{(4,5)}$. En el caso de los dientes inferiores estos factores corresponden a: el grosor de la cortical mandibular en comparación a la maxilar y la cercanía con el nervio alveolar inferior, si el diente se encuentra incluido, erupcionado o semierupcionado y la necesidad de realizar un colgajo, osteotomía y/u odontosección, el grado de impactación, la edad del paciente, experiencia del cirujano y tiempo de cirugía y las consideraciones anatómicas del diente ${ }^{(4-6)}$. Las complicaciones post extracción pueden ocurrir durante y/o 
después de la realización del acto quirúrgico. De acuerdo con el tiempo de evolución, estas serán inmediatas o postoperatorias. Las inmediatas afectan a piezas dentarias, tejidos blandos y tejidos duros, ocurriendo de manera intraoperatoria; las mediatas o postoperatorias, como alveolitis y hemorragias, por ejemplo, ocurren posteriormente a la extracción ${ }^{(7)}$. La etiología es variada, en muchos casos se debe a factores asociados a la técnica quirúrgica, estado de la pieza dentaria, o patología concurrente ${ }^{(7)}$. A pesar de ser un procedimiento rutinario, los pacientes han informado complicaciones que van entre $1 \%$ hasta $30,9 \%{ }^{(8-13)}$. Esta dispersión de los datos en los distintos estudios se puede explicar por causas metodológicas, derivadas del paciente y del tratamiento(14).

La complicación post extracción más frecuente es la alveolitis ${ }^{(15-20)}$ y ocurre con una frecuencia que varía desde el 0 al 35\% de todas las extracciones dentales ${ }^{(8)}$. Otras de las complicaciones más comúnmente reportadas son las hemorragias, parestesia del nervio mandibular, dolor e infecciones ${ }^{(5,21,22)}$

Actualmente, a pesar de que dos de las complicaciones posteriores a la exodoncia, la alveolitis y la hemorragia, sean patologías cubiertas por las Garantías Explícitas en Salud, específicamente por las Urgencias Odontológicas Ambulatorias, no existe un registro de la frecuencia de complicaciones posteriores a la extracción dentaria y cirugía de dientes incluídos en la población chilena. A partir de esto, y del desconocimiento en que ocurren complicaciones postquirúrgicas en otros procedimientos de cirugía bucal en nuestra población, nuestro objetivo principal fue determinar la incidencia de complicaciones posteriores a cirugía bucal en pacientes mayores de 12 años atendidos en el hospital San Borja-Arriarán de Santiago de Chile. Los objetivos secundarios corresponden a realizar un análisis descriptivo en relación a las características de los pacientes incluidos, describir la tasa de complicaciones según el procedimiento de cirugía bucal realizada, describir los distintos tipos de complicaciones y la incidencia según el tipo de procedimiento.

\section{METODOLOGÍA}

La investigación realizada corresponde a un estudio descriptivo y exploratorio que reporta la frecuencia y distribución de las complicaciones posteriores a procedimientos de cirugía bucal realizados en la Unidad de Cirugía Máxilofacial del Complejo Hospitalario San Borja-Arriarán, en Santiago de Chile a partir del registro de la casuística completa de doce meses de estudio, entre abril 2017 y marzo 2018. Para llevar a cabo esta investigación se obtuvo previamente la aprobación del Comité de Ética del Complejo Hospitalario San Borja-Arriarán y del Servicio de Salud Metropolitano Central. Participaron todos los cirujanos dentistas de la unidad.

Se seleccionaron todos los pacientes con indicación de cirugía bucal, que aprobaron su participación en el estudio a través de un consentimiento informado y que cumplieron con todos los criterios de selección definidos a continuación:

- Criterios de inclusión: Pacientes mayores de 12 años, sistémicamente sanos o con patologías controladas.

\section{- Criterio de exclusión}

- Pacientes alérgicos o con contraindicación de uso de paracetamol o antiinflamatorios no esteroidales.

- Pacientes que hayan sido sometidos a tratamiento antibiótico al menos 30 días antes de la cirugía.

- Pacientes con historia de pericoronaritis hasta 7 días antes de la intervención.

- Pacientes con enfermedades psiquiátricas que requieren de procedimientos especiales, como sedación o anestesia.

- Pacientes inmunocomprometidos.

- Pacientes que no asistieron a la cita de control o que no contestaron llamadas de control.

Todas las cirugías fueron realizadas con instrumental quirúrgico debidamente esterilizado y cumpliendo con los requerimientos sanitarios del Ministerio de Salud. Los cirujanos realizaron lavado de manos quirúrgico por 4 minutos con jabón gel de gluconato clorhexidina al $2 \%$, y usaron guantes estériles. Las cirugías fueron realizadas bajo anestesia locoregional. Se entregaron y explicaron las indicaciones postoperatorias tanto de forma oral como por escrito. Se les recomendó a los operadores utilizar paracetamol de un gramo cada ocho horas por tres días en procedimientos simples y sumar otro antiinflamatorio no esteroidal para procedimientos moderados o complejos.

El paciente fue citado a control a los 7 días para ser evaluado y llamado telefónicamente a los 30 días. En caso de complicaciones, estas fueron atendidas en el momento que el paciente consultó. Los parámetros clínicos fueron evaluados por dos recolectores de datos quienes fueron cirujanos dentistas entrenados y en los diagnósticos de complicaciones, los cuales fueron asesorados por cirujanos maxilofaciales que no participaron de la cirugía del paciente evaluado. Estos datos se registraron en el formulario de registro de datos confeccionado para este estudio. Se consideró la presencia o ausencia de complicaciones postoperatorias las cuales fueron determinadas a través del criterio clínico de los recolectores de datos entrenados.

La presencia de alveolitis fue definida en el examen clínico cuando se observó desintegración o ausencia del coágulo asociado a dolor moderado o intenso (sobre 4 en la escala visual analógica, de 0 a 10) después de las 48 horas de la intervención. El diagnóstico de hemorragia post extracción fue definido como el sangrado del alvéolo después de efectuada la exodoncia y que persistió, a pesar de las medidas hemostáticas realizadas en primera instancia, por el odontólogo y por el paciente.

Las variables independientes fueron consideradas con respecto al paciente como edad, sexo, enfermedades sistémicas, hábito tabáquico y consumo de alcohol o marihuana, al operador y su experticia como cirujano dentista, residente de la especialidad de cirugía máxilofacial o cirujano máxilofacial, tiempo quirúrgico (0 a 15 minutos, 16 a 30 minutos y 31 y más minutos) y variables de la cirugía como la dificultad quirúrgica según si fue leve, moderada o alta. Definiendo como moderadas aquellas en las que fue necesaria la realización de un colgajo y de dificultad alta los procedimientos en los que además se realizó osteotomía y/u odontosección. También se consideró si existió uso de medicación antibiótica como profilaxis antibiótica o antibioterapia posterior por indicación odontológica.

Análisis estadístico: Se realizó un análisis descriptivo de la información. La incidencia de complicaciones posterior a cirugía bucal se calculó utilizando tasas de forma general, por tipo de procedimiento y según el tipo de complicación. Se consideró para el cálculo de la incidencia de las complicaciones posteriores en cirugía bucal, el total de eventos en el numerador y el total de procedimientos incluídos como denominador. Para el cálculo de la incidencia de complicaciones según tipo de procedimiento, se utilizó el número de complicaciones para cada uno de ellos en el numerador y el total de procedimientos de cada tipo en el denominador. Todas las tasas presentadas se describieron en porcentajes. Para el cálculo de la incidencia de complicaciones según tipo de procedimiento, el numerador fueron las complicaciones asociadas para cada uno de ellos y el denominador fue el total de procedimientos de cada tipo. Todos los análisis fueron realizados utilizando el programa Stata/SE versión 14.0

Este estudio fue realizado mediante la declaración de la iniciativa STROBE para la confección de estudios observacionales ${ }^{(22)}$.

\section{RESULTADOS}

Durante los doce meses de estudio se realizaron 726 procedimientos de cirugía bucal, de éstos un total de 134 no cumplían con los criterios de selección y 60 fueron pacientes que no acudieron a la cita de control ni contestaron las llamadas telefónicas. Estos pacientes fueron excluidos del estudio y el número total analizado alcanzó 532 procedimientos de cirugía bucal.

La incidencia de complicaciones posteriores a cirugía bucal de este estudio fue un $5,5 \%$, presentándose principalmente en procedimientos de tipo exodoncia.

La tabla 1 presenta el análisis descriptivo de las características de los pacientes incluídos. Del total de los pacientes un $67 \%$ eran mujeres, las cuales presentaron una incidencia de complicaciones de un 5,3\%. Por otro lado, los hombres representan un $33 \%$ del total de pacientes incluídos y presentaron una incidencia de complicaciones de un $5,7 \%$. Un $35 \%$ de los pacientes tenía antecedente de alguna patología sistémica los cuales presentaron una incidencia de complicaciones de un $4,3 \%$, un $25 \%$ eran fumadores los cuales presentaron una incidencia de complicaciones de $5,3 \%$ y un $39 \%$ bebían alcohol los cuales presentaron una incidencia de complicaciones de un 5,8\%.

La tabla 2 detalla el porcentaje de incidencia para cada tipo de procedimiento en cirugía bucal. La cirugía de terceros molares presentó la mayor incidencia la cual corresponde a un $6,6 \%$ que representa un $4,5 \%$ del total de complicaciones. En segundo lugar se encuentran las enucleaciones de quistes, que presentaron una incidencia de un $33,3 \%$ que representa un $0,2 \%$ del total. Por último, la menor incidencia de complicaciones se presentó en las exodoncias simples con un $4,9 \%$ que representan un $0,8 \%$ de la incidencia total.

La tabla 3 describe los tipos de complicaciones postquirúrgicas y su incidencia según procedimiento. La complicación más frecuente fue la alveolitis, la cual presentó una incidencia de un $3,7 \%$ en exodoncias simples y de un $2,5 \%$ en exodoncia de terceros molares. La segunda complicación más frecuente fue el absceso de espacios faciales que se presentó solo en exodoncias de terceros molares con una incidencia de un 1,9\%. Luego la sigue la hemorragia con una incidencia de un $1,1 \%$ en 
Tabla 1: Características de los pacientes que presentaron complicaciones

\begin{tabular}{|c|c|c|}
\hline Variables & $\begin{array}{c}\mathrm{N}^{\circ} \text { de } \\
\text { procedimientos } \\
\text { / complicación } \\
\text { presente }\end{array}$ & $\begin{array}{l}\text { Incidencia por } \\
\text { grupo/ total (\%) }\end{array}$ \\
\hline Total & $532 / 29$ & $5,5 \%$ \\
\hline \multicolumn{3}{|l|}{ Sexo } \\
\hline Mujer & $357 / 19$ & $5,3 \% / 3,6 \%$ \\
\hline Hombre & $175 / 10$ & $5,7 \% / 1,9 \%$ \\
\hline \multicolumn{3}{|l|}{ Historia Médica } \\
\hline $\begin{array}{l}\text { Con antecedentes } \\
\text { mórbidos }\end{array}$ & $188 / 8$ & $4,3 \% / 1,5 \%$ \\
\hline $\begin{array}{l}\text { Sin antecedentes } \\
\text { mórbidos }\end{array}$ & $344 / 21$ & $6,1 \% / 3,9 \%$ \\
\hline Cirugía bucal previa & $350 / 16$ & $4,6 \% / 3 \%$ \\
\hline $\begin{array}{l}\text { Sin antecedentes } \\
\text { quirúrgicos }\end{array}$ & $182 / 13$ & $7,1 \% / 2,4 \%$ \\
\hline \multicolumn{3}{|l|}{ Hábitos } \\
\hline Tabaco & $132 / 7$ & $5,3 \% / 1,3 \%$ \\
\hline Alcohol & $207 / 12$ & $5,8 \% / 2,3 \%$ \\
\hline Marihuana & $55 / 1$ & $1,8 \% / 0,2 \%$ \\
\hline Sin hábitos & $138 / 9$ & $6,5 \% / 1,7 \%$ \\
\hline
\end{tabular}

Tabla 2: Complicaciones por tipo de procedimiento en cirugía bucal

\begin{tabular}{l|c|c}
\hline Tipo de procedimiento & $\begin{array}{c}\mathbf{N}^{\circ} \text { de } \\
\text { procedimientos } \\
\text { realizados/ } \\
\text { complicación } \\
\text { presente }\end{array}$ & $\begin{array}{c}\text { Incidencia } \\
\text { total/por } \\
\text { procedimiento } \\
\mathbf{( \% )}\end{array}$ \\
\hline Exodoncia terceros molares & $363 / 24$ & $4,5 \% / 6,6 \%$ \\
\hline Exodoncia & $81 / 4$ & $0,8 \% / 4,9 \%$ \\
\hline Exodoncia múltiple & $38 / 0$ & 0 \\
\hline Biopsias & $29 / 0$ & 0 \\
\hline Regularización de reborde & $6 / 0$ & 0 \\
\hline Otras cirugías & $8 / 0$ & 0 \\
\hline Enucleación de quistes & $3 / 1$ & $0,2 \% / 33,3 \%$ \\
\hline Frenectomías & $1 / 0$ & 0 \\
\hline Fenestraciones & $3 / 0$ & 0 \\
\hline Total & 532 & $5,5 \% /-$ \\
\hline
\end{tabular}

exodoncias de terceros molares, la equimosis ( 3 casos) y por último, la periostitis y hematoma ( 1 caso cada uno).

La tabla 4 describe la incidencia de complicaciones según las características del operador y del procedimiento. En relación a las características del operador, la mayor incidencia de complicaciones fueron en los procedimientos realizados por los residentes de la especialidad de cirugía maxilofacial (incidencia de un $7,4 \%$ ) y en los procedimientos que tuvieron un tiempo quirúrgico entre 16 a 30 minutos (incidencia de un 6,9\%). En relación a las caracteristicas del procedimiento, la mayor incidencia de complicaciones se dio en procedimientos de dificultad quirúrgica alta $(8,5 \%)$ y en procedimientos que no recibieron ningún tipo de medicación antibiótica $(5,7 \%)$.

\section{DISCUSIÓN}

El resultado observado en relación a la incidencia de complicaciones se sitúa dentro del amplio rango existente en los reportes de la literatura referente, ya que estos fluctúan entre un $1 \%$ a un $30 \%{ }^{(8-13)}$. Si bien no hay documentación de otros estudios equivalentes al presente, es posible hacer comparaciones con investigaciones cuyas metodologías y objetivos son similares. Bajo circunstancias semejantes, Bachmann ${ }^{(14)}$, en el
Tabla 3: Tipos de complicaciones postquirúrgicas y su incidencia según procedimiento quirúrgico

\begin{tabular}{l|c|c|c}
\hline $\begin{array}{c}\text { Tipo de } \\
\text { complicación }\end{array}$ & $\begin{array}{c}\mathbf{N}^{\circ} \text { Exodoncias } \\
\text { (\% incidencia) }\end{array}$ & $\begin{array}{c}\mathbf{N}^{\circ} \text { Exodoncias } \\
\text { de terceros } \\
\text { molares (\% } \\
\text { incidencia) }\end{array}$ & $\begin{array}{c}\mathbf{N}^{\circ} \\
\text { Enucleación } \\
\text { de quistes (\% } \\
\text { incidencia) }\end{array}$ \\
\hline $\begin{array}{l}\text { Sin } \\
\text { complicación }\end{array}$ & $77(95 \%)$ & $339(93,4 \%)$ & $2(66,7 \%)$ \\
\hline $\begin{array}{l}\text { Alveolitis } \\
\text { Húmeda }\end{array}$ & $3(3,7 \%)$ & $9(2,5 \%)$ & 0 \\
\hline Hemorragia & 0 & $4(1,1 \%)$ & 0 \\
\hline $\begin{array}{l}\text { Absceso } \\
\text { espacios } \\
\text { faciales }\end{array}$ & 0 & $7(1,9 \%)$ & 0 \\
\hline Parestesia & 0 & $1(0,3 \%)$ & 0 \\
\hline Equimosis & $1(1,2 \%)$ & $2(0,6 \%)$ & 0 \\
\hline Hematoma & 0 & 0 & $1(33,3 \%)$ \\
\hline Periostitis & 0 & $1(0,3 \%)$ & 0 \\
\hline Total & $81(100 \%)$ & $363(100 \%)$ & $3(100 \%)$ \\
\hline
\end{tabular}

Tabla 4: Incidencia de complicaciones según las características del procedimiento y operador

\begin{tabular}{|c|c|c|}
\hline Variables & $\begin{array}{l}\text { Total procedimientos } \\
/ \mathrm{N}^{\circ} \text { casos con } \\
\text { complicación }\end{array}$ & $\begin{array}{c}\text { Incidencia por grupo/ } \\
\text { total (\%) }\end{array}$ \\
\hline Total & $532 / 29$ & $5,5 \%$ \\
\hline \multicolumn{3}{|l|}{ Del Operador } \\
\hline \multicolumn{3}{|l|}{ Experiencia } \\
\hline Dentista & $162 / 6$ & $3,7 \% / 1,1 \%$ \\
\hline Becado & $189 / 14$ & $7,4 \% / 2,6 \%$ \\
\hline Máxilofacial & $181 / 9$ & $5 \% / 1,7 \%$ \\
\hline \multicolumn{3}{|c|}{ Tiempo Quirúrgico } \\
\hline 0 a $15 \mathrm{~min}$ & $259 / 11$ & $4,3 \% / 2,1 \%$ \\
\hline 16 a $30 \mathrm{~min}$ & $174 / 12$ & $6,9 \% / 2,3 \%$ \\
\hline$\geq$ a $31 \mathrm{~min}$ & $99 / 6$ & $6,1 \% / 1,1 \%$ \\
\hline \multicolumn{3}{|c|}{ Del Procedimiento } \\
\hline \multicolumn{3}{|c|}{ Dificultad Quirúrgica } \\
\hline Leve & $166 / 7$ & $4,2 \% / 1,3 \%$ \\
\hline Moderada & $142 / 3$ & $2,1 \% / 0,6 \%$ \\
\hline Alta & $224 / 19$ & $8,5 \% / 3,6 \%$ \\
\hline \multicolumn{3}{|c|}{ Medicación antibiótica } \\
\hline Profilaxis ATB & $23 / 1$ & $4,4 \% / 0,2 \%$ \\
\hline Antibioterapia & $33 / 1$ & $3 \% / 0,2 \%$ \\
\hline Sin medicación & $476 / 27$ & $5,7 \% / 5,1 \%$ \\
\hline
\end{tabular}

servicio maxilofacial del Hospital Base de Valdivia, con una muestra de 679 pacientes sanos con indicación de exodoncia de terceros molares, obtuvieron una prevalencia total de complicaciones de $11 \%$ y $2,4 \%$ en el caso de la alveolitis, tasa muy cercana a la obtenida en este análisis. La diferencia con respecto a la ocurrencia total de complicaciones puede deberse a la definición que existe sobre estos eventos, ya que, para estos autores, el edema, dolor y trismus son considerados complicaciones postquirúrgicas, en cambio, en este estudio, estas situaciones fueron consideradas esperables dentro del contexto de un proceso inflamatorio que resulta inevitable tras una cirugía bucal. Un estudio con resultados similares a los descritos en esta investigación es el realizado por Bui et al ${ }^{(13)}$ quienes con 583 pacientes sometidos a cirugía de extracción de terceros molares, obtuvieron un $4,6 \%$ total de complicaciones y $3,4 \%$ de alveolitis. De manera favorable, podemos comparar los resultados 
con el estudio de Eshghpour ${ }^{(24)}$ con una muestra de 256 procedimientos quirúrgicos de extracción de terceros molares impactados donde observaron una prevalencia que alcanzó un $19,14 \%$ de alveolitis. No obstante, esta diferencia favorecedora podría deberse a la complejidad de los procedimientos incluidos, ya que en el estudio citado se contemplaron sólo terceros molares impactados, en cambio, el diseño de este estudio incluyó cirugías con niveles de complejidad quirúrgica equivalentes y también de menor grado. Otro estudio prospectivo similar fue el realizado por Chuang ${ }^{(25)}$ con un total de 4.004 pacientes sometidos a cirugía de extracción de terceros molares observaron una prevalencia de complicaciones de $18,3 \%$ y $7,4 \%$ en el caso de alveolitis.

Al considerar la incidencia de alveolitis de forma aislada a las otras complicaciones postquirúrgicas, se observa que el procedimiento con mayor frecuencia de este evento son las exodoncias de dientes distintos a terceros molares. Para este tipo de intervención la incidencia alcanzó un $3,7 \%$. Cabe destacar que este tipo de procedimiento fue mayormente realizado por los operadores con menor grado de experiencia del servicio analizado, quienes eran cirujanos dentistas. Autores como Halabi ${ }^{(16)}$ sostienen la influencia que tiene la experiencia del operador en el desarrollo de alveolitis, ya que aquellos operadores con mayor práctica tendrían técnicas más prolijas y llevadas a cabo en tiempos menores.

En relación a la equimosis, los resultados reportados son muy similar al obtenido por Bachmann ${ }^{(14)}$ que alcanzó un $0,7 \%$.

Los casos de abscesos de espacios faciales fueron mayores a los obtenidos en otros estudios, como el realizado por Bui et al(13) quienes, con 583 pacientes sometidos a cirugía de extracción de terceros molares, observaron infección postoperatoria en un $0,8 \%$ de los casos. No obstante, Kaczmarzyk ${ }^{(26)}$ ha descrito que la ocurrencia de este desenlace va desde un 1 a un $15 \%$. Esta diferencia y amplio rango puede deberse a la libre definición utilizada en los distintos estudios.

La incidencia de la hemorragia suele ser baja y los resultados obtenidos se encuentran conformes a lo reportado. Bui et al ${ }^{(13)}$ observó hemorragias postoperatorias con una incidencia de $0,6 \%$, Bachmann ${ }^{(14)}$ un $0,3 \%$ y de manera aún menor, Chuang ${ }^{(25)}$ reportó un $0,1 \%$ de ocurrencia de estos eventos.

Los casos de parestesia se pueden comparar de manera favorable a los resultados obtenidos por Chuang ${ }^{(25)}$ quien reportó una incidencia del $1,6 \%$ y resultan consistentes con respecto a lo publicado por Bui et al ${ }^{(13)}$ con un $0,4 \%$ y a Bachmann ${ }^{(14)}$ quien observó estos eventos en un $0,3 \%$. La incidencia de hematoma es muy similar a lo observado por Bui ${ }^{(13)}$ quien reportó un $0,3 \%$ de estos eventos en cirugías de terceros molares. En este estudio, esta complicación ocurrió de manera postoperatoria a una enucleación y apicectomía de un diente maxilar. Esto podría explicarse por la avanzada edad del paciente involucrado, con 72 años, y a la mayor fragilidad capilar que sufren las personas en edades avanzadas ${ }^{(2)}$.

La principal ventaja de este estudio es que se realizó un registro de manera prospectiva mediante un formulario de registro de datos previamente establecido, el cual evita registros imprecisos como podrían presentarse en diseños retrospectivos. Como limitaciones podemos mencionar la distribución poco equitativa en cuanto a los tipos de procedimientos, considerando que la gran mayoría de estos fueron exodoncias hace difícil obtener resultados concluyentes con respecto a la incidencia de complicaciones postoperatorias en otros tipos de intervenciones en cirugía bucal.

\section{CONCLUSIONES}

Los resultados obtenidos muestran una incidencia de complicaciones postoperatorias en el Hospital Clínico San Borja-Arriarán de cirugía bucal en pacientes mayores de 12 años de un 5,5\%, siendo similar a lo reportado en la literatura.

\section{DECLARACIÓN DE CONFLICTO DE INTERÉS}

Los autores declaran no tener conflictos de interés.

\section{FUENTE DE FINANCIAMIENTO}

Financiado por Fondo para la investigación en odontología 17-002 (FIOUCh) Facultad de Odontología Dirección de Investigación Universidad de Chile

\section{RELEVANCIA CLÍNICA:}

En Chile las complicaciones posteriores a la exodoncia están incluidas en las Garantías Explícitas en Salud (GES). Sin embargo, no se conoce su incidencia a nivel nacional, así como también se desconoce la incidencia de complicaciones asociadas a otros procedimientos que engloba la cirugía bucal. Este estudio reporta una tasa de incidencia de un $5,5 \%$, concentrada principalmente en los procedimientos que involucran la exodoncia dentaria. Conocer las tasas de incidencia de estas complicaciones y las características de los pacientes afectados en nuestra realidad nacional nos permitirá tener una mejor aproximación clínica de los pacientes afectados.

\section{Bibliografía}

1. Gay Escoda C. Tratado de cirugía bucal. Madrid: Ergon; 1999. p. 392. 2. Valencia BAV, Marino A. Frecuencia de complicaciones post exodoncia simple. Oral. 2012;13(42):906-912

3. Rodrigues W, Okamoto R, Pellizer E, Carrijo A, Nazareno A. Antibiotic prophylaxis for third molar extraction in healthy patients: Current scientific evidence. Quintessence Int. 2015;2(46):149-161.

4. Susarla SM, Dodson TB. Risk factors for third molar extraction difficulty. J Oral Maxillofac Surg. 2004;62(11):1363-1371.

5. Blondeau F, Daniel N. Extraction of impacted mandibular third molars: postoperative complications and their risk factors. J Can Dent Assoc. 2007;73(4):325

6. Llerena G, Arrascue M. Tiempo de cirugía efectiva en la extracción de los terceros molares realizadas por un cirujano oral y maxilofacial con experiencia. Rev Estomatol Herediana. 2006;16(1):40-45.

7. MINSAL, Guía Clínica Urgencias Odontológicas Ambulatorias. 2a. ed. 2011, Santiago: Ministerio de Salud.

8. Nusair YM, Younis MH. Prevalence, clinical picture, and risk factors of dry socket in a Jordanian dental teaching center. J Contemp Dent Pract. 2007;8(3):53-63.

9. Dolci E, Escoda GC, Arnabat J. La prevención de la alveolitis seca. Rev Eur Odonto- Estomatol. 1992; 5:261-270.

10. Bloomer CR. Alveolar osteitis prevention by immediate placement of medicated packing. Oral Surg Oral Med Oral Pathol Oral Radiol Endod. 2000;90(3):282-284

11. Heasman PA, Jacobs DJ. A clinical investigation into the incidence of dry socket. Br J Oral Maxillofac Surg. 1984;22(2):115-22.

12. Tarakji B, Saleh LA, Umair A, Azzeghaiby SN, Hanouneh S. Systemic review of dry socket: aetiology, treatment, and prevention. J Clin Diagn Res. 2015;9(4): ZE10-3.

13. Bui $\mathrm{CH}$, Seldin EB, Dodson TB. Types, frequencies, and risk factors for complications after third molar extraction. J Oral Maxillofac Surg. 2003;61(12):1379 138.

14. Bachmann H, Cáceres R, Muñoz C, Uribe S. Complicaciones en cirugía de terceros molares entre los años 2007-2010, en un hospital urbano, Chile. Int J Odontostomat. 2014;8(1):107-112.

15. Jaafar N, Nor GM. The prevalence of post-extraction complications in an outpatient dental clinic in Kuala Lumpur Malaysia--a retrospective survey. Singapore Dent J. 2000;23(1):24-28.
16. Halabí D, Escobar J, Muñoz C, Uribe S. Logistic regression analysis of risk factors for the development of alveolar osteitis. J Oral Maxillofac Surg. 2012;70(5):1040-4 17. Figueiredo R, Valmaseda-Castellón E, Berini-Aytés L, Gay-Escoda C. Delayedonset infections after lower third molar extraction: a case-control study. J Oral Maxillofac Surg. 2007;65(1):97-102.

18. Dyer TA. A five-year evaluation of an NHS dental practice-based specialist minor oral surgery service. Community Dent Health. 2013;30(4):219-226.

19. Venkateshwar GP, Padhye MN, Khosla AR, Kakkar ST. Complications of exodontia: a retrospective study. Indian J Dent Res. 2011 Sep-Oct;22(5):633-8.

20. Yengopal V, Mickenautsch S. Chlorhexidine for the prevention of alveolar osteitis. Int J Oral Maxillofac Surg. 2012;41(10):1253-64.

21. Pitekova L, Satko I, Novotnakova D. Complications after third molar surgery. Bratisl Lek Listy. 2010;111(5):296-8.

22. Woldenberg Y, Gatot I, Bodner L. latrogenic mandibular fracture associated with third molar removal. Can it be prevented? Med Oral Patol Oral Cir Bucal. 2007;12(1):E70-2

23. Von Elm E, Altman DG, Egger M, Pocock SJ, Gøtzsche PC, Vandenbroucke JP, STROBE Initiative. [The Strengthening the Reporting of Observational Studies in Epidemiology (STROBE) statement: guidelines for reporting of observational studies]. Internist (Berl). 2008 Jun;49(6):688-93.

24. Eshghpour $M$, Nejat $A H$. Dry socket following surgical removal of impacted third molar in an Iranian population: incidence and risk factors. Niger $\mathrm{J}$ Clin Pract. 2013;16(4):496-500.

25. Chuang SK, Perrott DH, Susarla SM, Dodson TB. Age as a risk factor for third molar surgery complications. J Oral Maxillofac Surg. 2007;65(9):1685-92.

26. Kaczmarzyk T, Wichlinski J, Stypulkowska J, Zaleska M, Panas M, Woron J. Single-dose and multi-dose clindamycin therapy fails to demonstrate efficacy in preventing infectious and inflammatory complications in third molar surgery. Int $J$ Oral Maxillofac Surg. 2007;36(5):417-22 